\title{
Optimal charging of electric vehicles in microgrids through discrete event optimization
}

\author{
G. Ferro, F. Laureri, R. Minciardi, Member, IEEE and M. Robba, Member, IEEE
}

\begin{abstract}
In this paper, a discrete event approach is proposed for the optimal charging of electrical vehicles in microgrids. In particular, the considered system is characterized by renewable energy sources (RES), non-renewable energy sources, electrical storage, a connection to the external grid and a charging station for electric vehicles (EVs). The decision variables are relevant to the schedule of production plants, storage systems and EVs' charging. The objective function to be minimized is related to the cost of purchasing energy from the external grid, the use of nonrenewable energy sources and tardiness of customer's service. The proposed approach is applied to a real case study and it is shown that it allows to considerably reduce the dimension of the problem (and thus the computational time required) as compared to a discrete-time approach.
\end{abstract}

Index Terms - microgrids, scheduling, discrete event control, optimization.

\section{INTRODUCTION AND STATE OF THE ART}

Electric vehicles (EV) are more and more popular due to the fewer emissions and lower oil dependency [1]. World organizations are encouraging national authorities to support the spread of electric or hybrid vehicles. Mass deployment of EVs could be a good solution, but, unfortunately, may cause technical problems to the electrical grid due to intermittent and distributed loads [2]. In fact, the available energy might become insufficient to host the recharge processes of too many EVs. Moreover, the adoption of many renewable energy source increases the uncertainty and intermittency of the power production, leading to possible problems to the grid.

A solution to avoid these problems is to integrate the charging station facilities within smart microgrids [3]. EVs tend to be treated as a new form of the mobile energy storage system with the potentiality to promote energy management in microgrids. In this framework, in [4] a day-ahead EV charging scheduling based on game theory is proposed. The impacts of the EVs demand on the electricity prices are formulated via a game model in the scheduling, considering possible actions of other EVs. In [5], a cooperative optimization method for capacity configuration and economic dispatch of EVs in microgrids considering time and space varying energy prices is presented. The charging of a large number of vehicles in the same node of the grid is a challenging problem: it is necessary to respect the maximum power of the specific node while serving in a reasonable time the customers, and to define new optimization problems and new solution approaches to obtain results in a reasonable runtime. In [6], the authors develop a distributed algorithm based on alternating direction method of multipliers to decompose the problem. Scalability and effectiveness of this approach are tested in the IEEE 119-bus and IEEE 906-bus distribution networks. The literature also analyses the possibility of integrating the charging station of EVs in parking lots. In particular, in [7] the authors propose a new bilevel framework for operational scheduling of a smart distribution company with EV parking lot and renewable energy sources. In the proposed bi-level model, maximization of the company's profit is obtained in the upper-level problem by minimizing the cost of power purchased from the wholesale market. The lower-level problem aims to maximize the profit of the parking lot owner. This model is converted to a non-linear single-level problem by using Karush-KuhnTucker conditions.

In the most recent literature even event driven scheduling approaches for EVs are discussed, just like in [8] and [9]. However, all event driven approaches are essentially on-line scheduling algorithms aiming to "correct" a pre-existing scheduling when some "event" like the arrival of a new customer (vehicle) occurs. The main difference with our approach is that the optimal scheduling of a given set of jobs (vehicles) is here considered. Other works based on discrete event approaches are present in the literature of energy management but only from a simulation point of view [10].

In a previous work [11], a discrete time approach is proposed for a similar optimization problem: however, the high number of variables required a high computational time. As can be seen in the following, the approach proposed in the present paper reduces significantly the computational burden with respect to a discrete time approach. However, the adoption of a discrete event approach requires the introduction of some hypotheses concerning the way the control strategy is developed and applied to the system.

The considered problem falls within the class of scheduling problems [12], since the objective is to assign the optimal timing of EVs charging process. It is worth noting that for non-regular optimization objectives the timing decisions are not trivial. Non-regular optimization objectives are those objectives for which an advantage may be obtained by delaying the completion time of a job while letting the completion times of all other jobs unaltered [13]. In the following, it will be shown that the cost to be minimized in

\footnotetext{
* G. Ferro, F. Laureri, R. Minciardi and M. Robba are with Department of Informatics, Bioengineering, Robotics, and Systems Engineering University of Genova Italy (e-mail giulio.ferro@edu.unige.it)
} 
this paper generally falls within the class of non-regular objectives.

The formalization of the problem presented in this paper aims at optimizing the timing of the execution of jobs (the charging of the various vehicles) on a single resource (the charging station), assuming that the service sequence is given and coincident with the arrival sequence of the vehicles. In this way, the dynamics of the considered system may be represented within a discrete event setting.

The paper is organized as follows. In the next section, the discrete event approach is presented. In the third section, the optimization problem is formalized, in connection with a specific system model. In the fourth, the results of the application to a real case study is presented. Some concluding remarks are finally provided in the last section.

\section{THE SYSTEM MODEL}

In this paper, a discrete event approach is applied to the optimal scheduling of the plants of a microgrid. The choice of a discrete event representation is highly recommended in order to limit the number of decision variables and thus to reduce the runtime for the solution of the optimization problem [10]. In fact, in order to keep the problem tractable, it is necessary, in discrete time modelling, to limit the number of the time intervals. This can be obtained either by reducing the length of the optimization horizon or increasing the length of the time discretization intervals. Of course, both these choices are in general not satisfying. For these reasons, it is highly preferable to adopt a discrete event approach, that tracks events when they take place, without adopting any type of approximation.

The system is described by a sequence of state transitions induced by the events that affect the system behaviour (an iteration of the state equation takes place only in coincidence with the occurring of an event).

The system taken into account for this paper is composed of the following elements (see Figure 1):

- A traditional (non-renewable) energy source;

- A renewable resource that is intermittent and not controllable. It is assumed that a forecast of the energy coming from the renewable source is available for the whole horizon of interest;

- A point of common coupling (PCC) with the main grid, that guarantees a bidirectional power flow from/to the microgrid;

- An electrical storage element;

- A single charging station for electric vehicles; that can serve a single vehicle at a time (pre-emption is excluded);

- A traditional electric load characterized by a known daily pattern.

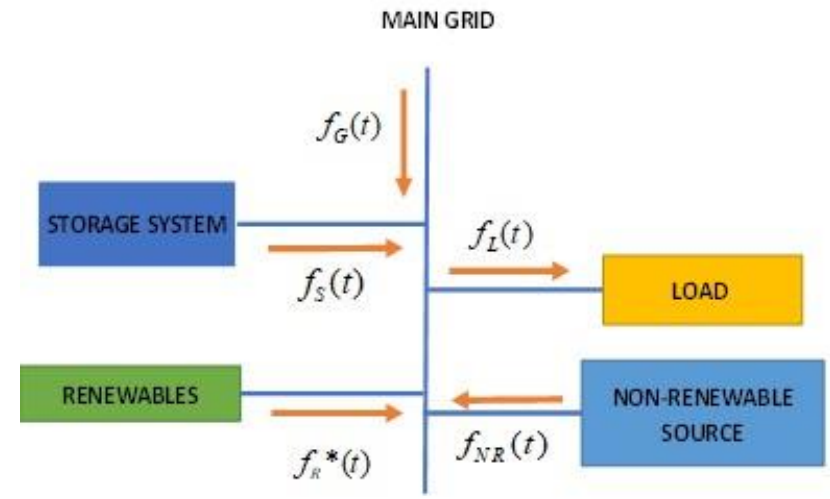

Figure 1. The considered microgrid.

For each vehicle $V_{i}$ it is assumed that the following information is known a priori:

- Release time $\left(r t_{i}\right)$ : the time when the vehicle becomes available for service;

- Dead line $\left(d l_{i}\right)$ : the time at which the service must be completed (hard requirement);

- Due date $\left(d d_{i}\right)$ : the time at which the service for the vehicle should be completed (soft requirement); ;

- Energy request $\left(E r_{i}\right)$ : the energy required to charge vehicle $V_{i}$

- Penalty coefficient $\alpha_{i}$ for unitary tardiness: the cost paid for a unit delay, for vehicle $V_{i}$. It is expressed in $[€ /(\mathrm{h}$ $\mathrm{kWh}$ )].

It is assumed that the service cannot be refused to any customer and that deadline constraints must be fulfilled.

The power flows, expressed as functions of time instant $t$, are:

- $f_{L}(t)$ : the power flow from the charging station to the vehicle (no vehicle to grid is allowed);

- $f_{N L}(t)$ : the net load defined as the difference $f_{N L}^{*}(t)-f_{R}^{*}(t)$, where $f_{N L}^{*}(t)$ is the non-deferrable load, and $f_{R}^{*}(t)$ is the power coming from renewables;

- $f_{G}(t)$ : the power flow from/to the main grid (active sign convention is used) ;

- $f_{N R}(t)$ : the power generated by the traditional source;

- $f_{S}(t)$ : the power flow from/to the storage unit (active sign convention is used).

In addition, the value of the energy level of the storage at time instant $t$ will be denoted by $x(t)$. It has been already pointed out that the service sequence is assumed to be given, and that the vehicles are charged following the order of their arrivals. Thus, only timing decisions have to be taken. Since in this model the energy request $E R_{i}$ by each vehicle has to be completely satisfied, the possible customer's dissatisfaction is quantified only by a tardiness cost. 
Figure 2 represents the time intervals between the completion of two services of the $(i-1)$-th vehicle, namely $C_{i \text { - }}$ ${ }_{1}$, and the completion time of the $i$-th vehicle, $C_{i}$, can be partitioned in two different time intervals:

- $\quad \mathrm{CH}_{i}$, the charging time (interval) for the $i$-th vehicle;

- $\quad I D L E_{i}$, the idle time interval before the charging of the $i$-th vehicle.

It is assumed that $I D L E_{i}$ must be greater than or equal to a minimum value $\varepsilon$, that is the set-up time of the charging station.

The values of interest of the state variable $x(t)$ (it is assumed that $x(0)$ is known) are those corresponding to the completion of each service.

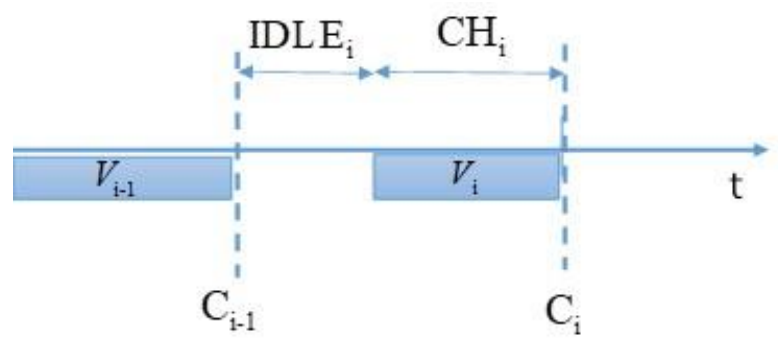

Figure 2. The time intervals between two successive completion time instants.

The following restrictive assumptions (but not too unrealistic) are introduced in order to obtain a finite-dimensional optimization problem (that is a parameter optimization problem instead of a functional optimization problem):

- the power flow $f_{G}(t)$, and the power injected by the nonrenewable source $f_{N R}(t)$ are kept constant within each idle time interval $\left(\mathrm{C}_{i-1}, \mathrm{C}_{i}-\mathrm{CH}_{i}\right)$ and at another constant value within each charging time interval

$\left(C_{i-1}+I D L E_{i}, C_{i}\right)$, for $i=1, \ldots, N$, being $C_{0}$ the initial time instant $\left(C_{0}=0\right)$;

- the power flow to the charging station $f_{L}(t)$ is kept constant within each time interval. This constant value will be denoted as $f_{\mathrm{L}, i} i=1, \ldots, N$.

- The forecasts of the renewable power $f_{R}^{*}(t)$, the nondeferrable load $f_{N L}^{*}(t)$ and the selling/buying prices $(\operatorname{SP}(t)$ and $B P(t))$ to/from the main grid are not affected by uncertainties.

The system state equation can be written by means of a discrete event recursion as

$$
x\left(C_{i}\right)=x\left(C_{i-1}\right)-\bar{f}_{S 1, i} I D L E_{i}-\bar{f}_{S 2, i} C H_{i} \quad i=1, \ldots, N
$$

where $\bar{f}_{S 1, i}$ is the average value of $f_{S}(t)$ within time interval $\left(C_{i-1}, C_{i-1}+I D L E_{i}\right), \bar{f}_{S 2, i}$ is the average value of $f_{S}(t)$ within time interval $\left(C_{i-1}+I D L E_{i}, C_{i}\right)$. Namely,

$$
\begin{array}{ll}
\bar{f}_{S 1, i}=\frac{1}{I D L E_{i}} \int_{C_{i-1}}^{C_{i-1}+I D L E_{i}} f_{S}(t) d t & i=1, \ldots, N \\
\bar{f}_{S 2, i}=\frac{1}{C H_{i}} \int_{C_{i-1}+I D L E_{i}}^{C_{i}} f_{S}(t) d t & i=1, \ldots, N
\end{array}
$$

For the sake of brevity, in the following $x\left(C_{i}\right)$ will be denoted simply as $x_{i}$.

In this work, the power network is considered as a single busbar, i.e. no grid topology is taken into account. This assumption is widely used in the literature regarding small microgrids [12]. Thus, the power balance equation is given by

$f_{L}(t)=f_{G}(t)+f_{S}(t)-f_{N L}(t)+f_{N R}(t)$

that is a necessary condition for each time instant. As we are considering a discrete event formalization, (4) can be imposed only on the average values of the variables over time intervals. Thus, basing on the previous restrictive assumptions, we can write

$0=f_{G 1, i}+\bar{f}_{S 1, i}-\bar{f}_{N L 1, i}+f_{N R 1, i} \quad i=1, \ldots, N$

$f_{L, i}=f_{G 1, i}+\bar{f}_{S 2, i}-\bar{f}_{N L 2, i}+f_{N R 2, i} \quad i=1, \ldots, N$

where

$\bar{f}_{N L 1, i}=\frac{1}{I D L E_{i}} \int_{C_{i-1}}^{C_{i-1}+I D L E_{i}} f_{N L}(t) d t \quad i=1, \ldots, N$

$\bar{f}_{N L 2, i}=\frac{1}{C H_{i}} \int_{C_{i-1}+I D L E_{i}}^{C_{i}} f_{N L}(t) d t \quad i=1, \ldots, N$

Observe that, as the function $f_{N L}(t)$ is assumed to be known by forecasted data, the terms $\bar{f}_{N L 1, i}, \bar{f}_{N L 2, i}$ can be expressed as a function of $C_{i-1}, C_{i}, I D L E_{i}$, which are included within the set of the decision variables of the problem.

In the solution of the optimization problem that we are defining, only the values of $\bar{f}_{S 1, i}$ and $\bar{f}_{S 2, i}$ will be determined. These values will be given as set points to the internal control loop of the storage system, that takes into account also the continuous time constraints of the system.

\section{THE OPTIMIZATION PROBLEM}

The objective function considers multiple cost functions: the cost due to the energy production from non-renewable sources, the energy bought/sold from/to the main grid, and a penalty cost for tardiness. Note that costs related to energy have to be considered separately for idle and charging time intervals. Thus, the following minimization is sought

$\min \sum_{i=1}^{N}\left\{F G_{I D L E, i}+F G_{C H, i}+C_{N R} f_{N R 1, i} I D L E_{i}+C_{N R} f_{N R 2, i} C H_{i}\right.$ 
where

$$
\begin{aligned}
& F G_{I D L E_{i}}=\left\{\begin{array}{l}
{\left[\int_{C_{i-1}}^{C_{i-1}^{+I D L E}} B P(t) d t\right] f_{G 1, i}^{+}} \\
-\left[\int_{C_{i-1}}^{C_{i-1}+I D L E} S P(t) d t\right] f_{G 1, i}^{-}
\end{array}\right\} \quad i=1, \ldots, N \\
& F G_{C H_{i}}=\left\{\begin{array}{l}
{\left[\int_{C_{i-1}+I D L E_{i}}^{C_{i}} B P(t) d t\right] f_{G 2, i}^{+}} \\
-\left[\int_{C_{i-1}+I D L E_{i}}^{c_{i}} S P(t) d t\right] f_{G 2, i}^{-}
\end{array}\right\} \quad i=1, \ldots, N
\end{aligned}
$$

being $C_{N R}$ the unit cost $[€ / \mathrm{kWh}]$ for generation of nonrenewable energy, and $\alpha_{i}[€ / \mathrm{kWh} * \mathrm{~h}]$ the penalty per unit of tardiness and unit of energy request. Moreover, the following notation is used

$$
\begin{array}{rr}
f_{G 1, i}^{+}=\max \left\{f_{G 1, i}, 0\right\} & i=1, \ldots, N \\
f_{G 1, i}^{-}=\max \left\{-f_{G 1, i}, 0\right\} & i=1, \ldots, N \\
f_{G 2, i}^{+}=\max \left\{f_{G 2, i}, 0\right\} & i=1, \ldots, N \\
f_{G 2, i}^{-}=\max \left\{-f_{G 2, i}, 0\right\} & i=1, \ldots, N
\end{array}
$$

so that

$$
\begin{array}{lr}
f_{G 1, i}=f_{G 1, i}^{+}-f_{G 1, i}^{-} & i=1, \ldots, N \\
f_{G 2, i}=f_{G 2, i}^{+}-f_{G 2, i}^{-} & i=1, \ldots, N
\end{array}
$$

In the cost function (9), the first and second term in the summation, expressed by (10) and (11), represent the net cost for buying/selling energy from/to the main grid during the $i$ th idle and in the $i$-th charging time, respectively. The third and the fourth terms represent the energy cost from the nonrenewable source in the $i$-th idle time, and in the $i$-th charging time, respectively. Finally, the last term represents the charging process tardiness cost of the $i$-th vehicle.

It is noteworthy that in the cost function there is no term referring to the revenue for the charging services. That is justified by the assumption that all customers must be completely serviced. Note that, as $B P(t)$ and $S P(t)$ are assumed known, terms (10) and (11) can be expressed as functions of the decision variables $C_{i-1}, C_{i}, I D L E_{i}$.

Now, the proposed optimization problem can be formalized as the minimization in (9), subject to constraints (1), (5)-(8), (10), (11), (16), (17), along with the following further constraints have to be considered

$$
\begin{array}{ll}
C_{i} \leq d l_{i} & i=1, \ldots, N \\
C_{i}-C H_{i} \geq r t_{i} & i=1, \ldots, N \\
I D L E_{i} \geq \varepsilon & i=1, \ldots, N
\end{array}
$$

$$
\begin{array}{ll}
I D L E_{i}=C_{i}-C H_{i}-C_{i-1} & i=1, \ldots, N \\
f_{L, i} C H_{i}=E R_{i} & i=1, \ldots, N \\
\operatorname{tard}_{i} \geq C_{i}-d d_{i} & i=1, \ldots, N \\
-F_{g}^{M A X} \leq f_{G 1, i} \leq F_{g}^{M A X} & i=1, \ldots, N \\
-F_{g}^{M A X} \leq f_{G 2, i} \leq F_{g}^{M A X} & i=1, \ldots, N \\
x_{\text {Min }} \leq x_{i} \leq x_{M a x} & i=1, \ldots, N \\
-F_{S, \mathrm{MAX}} \leq \bar{f}_{S 1, i} \leq F_{S, \mathrm{MAX}} & i=1, \ldots, N \\
-F_{S, \mathrm{MAX}} \leq \bar{f}_{S 2, i} \leq F_{S, \mathrm{MAX}} & i=1, \ldots, N \\
f_{\mathrm{L}, \mathrm{MIN}} \leq f_{\mathrm{L}, i} \leq f_{\mathrm{L}, \mathrm{MAX}} & i=1, \ldots, N \\
f_{\mathrm{NR}, \text { Min }} \leq f_{\mathrm{NR} 2, i} \leq f_{\mathrm{NR}, \mathrm{MAX}} & i=1, \ldots, N \\
f_{\mathrm{NR}, \text { Min }} \leq f_{\mathrm{NR} 2, i} \leq f_{\mathrm{NR}, \mathrm{MAX}} &
\end{array}
$$

All the considered variables are non-negative, except $f_{G 1, i}, f_{G 2, i}$ and $\bar{f}_{S 1, i}, \bar{f}_{S 2, i}$.

. Constraints (18) imply the respect of the deadlines, whereas constraints (19) impose that the service cannot start before the release time. Constraints (20) impose a minimum duration of the idle time before a service, and constraints (21) are equivalent to the definition of idle times as functions of charging and completion times. Constraints (22) impose the satisfaction of the entire energy request for each vehicle.

Constraints (23) define the tardiness of the services. Note that $\operatorname{tard}_{i}$ is defined as

$$
\operatorname{tard}_{i}=\max \left\{C_{i}-d d_{i}, 0\right\}
$$

However, since the tardiness variables are constrained to be non-negative, and one of the terms in the minimization in (9) has the objective of minimizing the overall tardiness cost, the max in the definition may well be substituted by the inequality in constraints (23). Finally, technical constraints in (24)-(31) represent physical upper and lower bounds on the power flows and the energy contents in the system.

Some comments are needed about the structure of the optimization problem above defined. First, it is important to note that the problem is, in general, a nonlinear mathematical programming problem, since the definite integrals present in the cost function (9), as well as those in constraints (10) and (11), are nonlinear functions of the decision variables of the problem. Besides, there is also a product of decision variables in constraints (22). Second, the presence of a time-varying net non-deferrable load makes the considered problem nonregular, using the terminology of scheduling theory. In fact, it may be advantageous to delay the execution of a job by shifting it to a period with lower net non-deferrable energy demand. In a similar way, the presence of time-varying buying/selling prices, may make convenient the shifting of services to time periods with lower energy buying prices. Thus, even though the service sequence is assumed fixed, the 
timing problem is not trivial and requires the solution of a mathematical programming problem. Finally, observe that the statement of the problem has been provided in the assumption that the forecasts of the net non-deferrable load for the whole horizon of interest. These forecasts are retained as completely reliable, and thus, although in a discrete event setting, this gives rise to a sort of predictive control scheme. Then, as in any predictive control scheme, one can imagine the repeated application of the proposed approach at different time instants, conditioned by the most recent available information about the current system state and predictions about the future pattern of the net non-deferrable load. It can be reasonable, for instance, to think of solving a new problem at any service completion time instants.

\section{APPLICATION TO A CASE STUDY}

The case study chosen to test the proposed approach refers to a set of facilities in the Savona municipality (Italy). Six EVs $(N=6)$ are supposed to be charged in a grid-connected microgrid.

The nonlinear optimization problem defined by (1)-(31) has been solved through the use of Lingo optimization tool (www.lindo.com). Table 1 provides data of the elements of the microgrid.

TABLE I. SYSTEM PARAMETERS

\begin{tabular}{|c|c|c|c|}
\hline Parameter & Value & Parameter & Value \\
\hline$x_{\text {MAX }}$ & $125[\mathrm{kWh}]$ & $f_{\mathrm{L}, \mathrm{MIN}}$ & $10[\mathrm{~kW}]$ \\
\hline$x_{\text {Min }}$ & $40[\mathrm{kWh}]$ & $f_{\mathrm{NR}, \mathrm{MAX}}$ & $65[\mathrm{~kW}]$ \\
\hline$F_{g}^{\text {MAX }}$ & $451[\mathrm{~kW}]$ & $f_{\mathrm{NR}, \text { Min }}$ & $6.5[\mathrm{~kW}]$ \\
\hline$F_{S, \text { MAX }}$ & $36[\mathrm{~kW}]$ & $x(0)$ & $60[\mathrm{kWh}]$ \\
\hline$f_{\mathrm{L}, \mathrm{MAX}}$ & $50[\mathrm{~kW}]$ & $\varepsilon$ & $0.08[\mathrm{~h}]$ \\
\hline
\end{tabular}

In order to express the functions $\bar{f}_{N L 1, i}\left(C_{i-1}, C_{i}, I D L E_{\mathrm{i}}\right)$ and $\bar{f}_{N L 2, i}\left(C_{i-1}, C_{i}, I D L E_{\mathrm{i}}\right)$, a forecast of the net load $f_{N L}(t)$ is available for a complete day with a time discretization step of 15 minutes. To compute both functions, in order to calculate the integral functions (10) and (11), the forecasts has been interpolated via a seventh order polynomial function (Figure $3)$. In the considered case study, the electrical demand is much greater than renewables and thus the pattern follows the one of load but lowered by photovoltaics contribution.

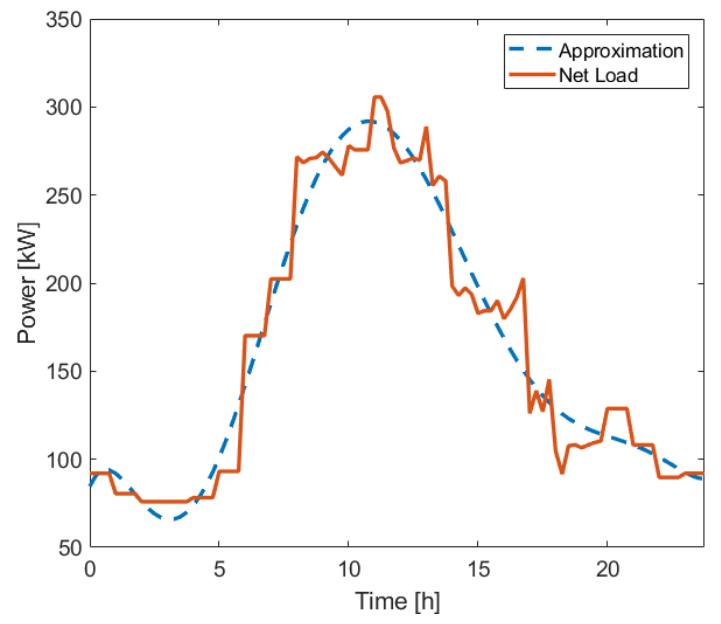

Figure 3: Net load function and its polynomial approximation.

Table 2 reports data for each vehicle. Numbers represent time instants expressed in hours, starting from an initial time instant put equal to 0 (corresponding to 8:00 a.m.).

TABLE II. THE PARAMETERS OF THE 6 VeHICLES

\begin{tabular}{|c|c|c|c|c|c|c|}
\hline Vehicle & $\mathbf{1}$ & $\mathbf{2}$ & $\mathbf{3}$ & $\mathbf{4}$ & $\mathbf{5}$ & $\mathbf{6}$ \\
\hline $\boldsymbol{r e l}_{\boldsymbol{i}}$ & 0 & 4 & 7.5 & 9.5 & 11 & 13 \\
\hline $\boldsymbol{d d}_{\boldsymbol{i}}$ & 3 & 5.6 & 10.5 & 11.5 & 13 & 15 \\
\hline $\mathbf{d l}_{\mathbf{i}}$ & 7 & 7 & 12 & 12 & 16 & 16 \\
\hline $\mathbf{E r}_{\mathbf{i}}[\mathrm{kWh}]$ & 28 & 30 & 25 & 20 & 18 & 25 \\
\hline $\boldsymbol{\alpha}_{\mathbf{i}}[€ /(\mathrm{h} \mathrm{kWh})]$ & 0.1 & 0.1 & 0.1 & 0.1 & 0.1 & 0.1 \\
\hline
\end{tabular}

For this test case it is assumed that the prices for purchasing or selling energy from/to the main grid are constant $(B P=0.15$ $[€ / \mathrm{kWh}]$ and $S P=0.12[€ / \mathrm{kWh}])$. All the vehicles considered have the same penalty coefficient for the tardiness, so no vehicle has any preference. Owing to the time-invariance of the prices, terms in (10) and (11) can be simply expressed as

$F G_{I D L E_{i}}=\left(B P f_{G 1, i}^{+}-S P f_{G 1, i}^{-}\right) I D L E_{i} \quad i=1, \ldots, N$

$F G_{C H_{i}}=\left(B P f_{G 2, i}^{+}-S P f_{G 2, i}^{-}\right) C H_{i} \quad i=1, \ldots, N$

The results obtained are reported in Table 3 and represented in the following Figures 4-6.

TABLE III. OPTIMAL SERVICES SCHEDULING

\begin{tabular}{|c|c|c|c|c|c|c|}
\hline Vehicle & $\mathbf{1}$ & $\mathbf{2}$ & $\mathbf{3}$ & $\mathbf{4}$ & $\mathbf{5}$ & $\mathbf{6}$ \\
\hline$C_{i}[\mathrm{~h}]$ & 5.26 & 6.34 & 10.5 & 11.24 & 12.92 & 13.83 \\
\hline$C_{i}[\mathrm{~h}]$ & 0.93 & 1 & 2.52 & 0.66 & 0.79 & 0.83 \\
\hline$I D L E_{i}[\mathrm{~h}]$ & 3.33 & 0.08 & 1.62 & 0.08 & 0.87 & 0.08 \\
\hline tard $_{i}$ & 2.26 & 0.74 & 0 & 0 & 0 & 0 \\
\hline
\end{tabular}




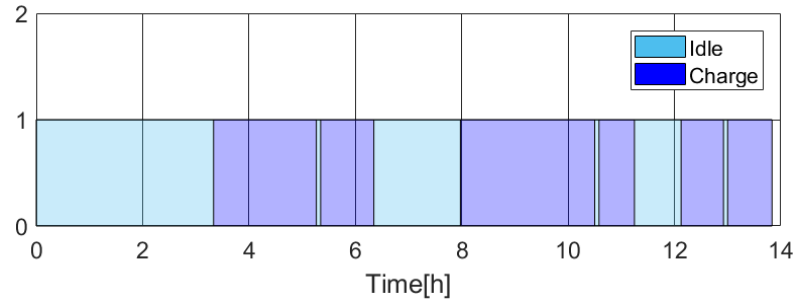

Figure 4. Gantt diagram of the sequence of the 6 services.

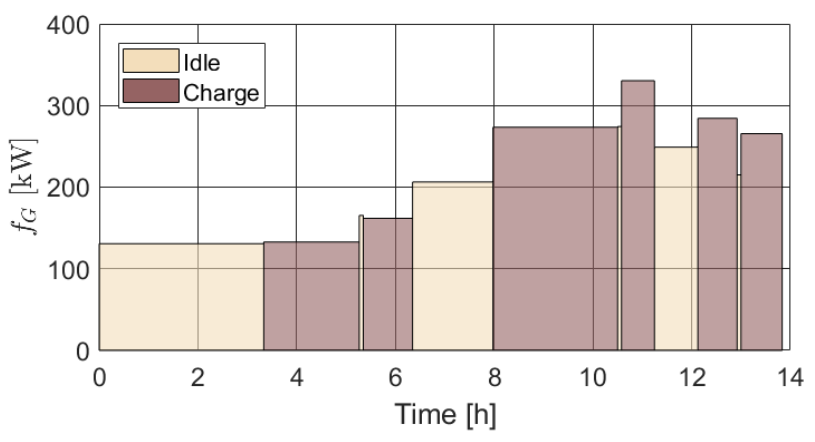

Figure 5. The power bought from the external grid

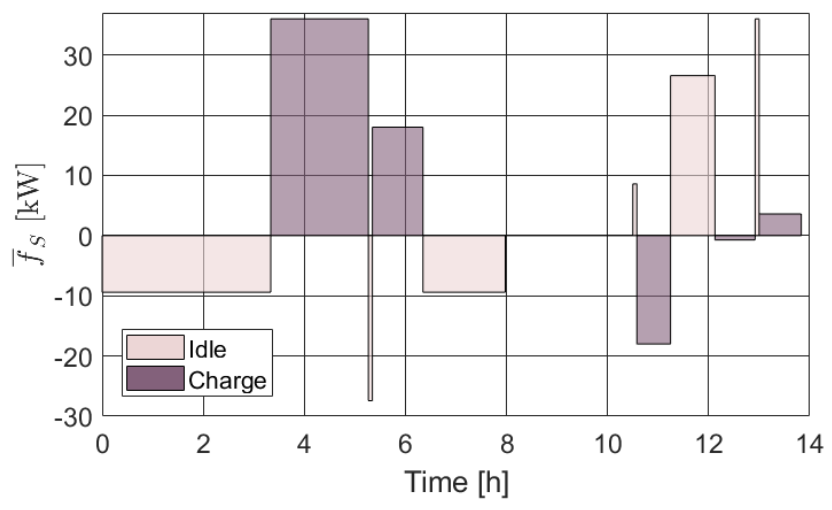

Figure 6. The (average) power flow from/to the storage

\section{Remark 1}

In order to appreciate the difference between the discreteevent approach proposed in this paper and the discrete-time approach proposed by the same authors in [11], let us consider two problem instances: a) a discrete-time instance [11], with 4 vehicles to be charged over 24 hours, b) a discrete-event instance with 6 vehicles over the same time horizon of 24 hours. In [11] it is reported that the problem instance gives rise to a mathematical programming problem with 945 integer variables and 240 continuous variables, whose solution by the commercial software LINGO requires a computational time approximately equal to 6 hours. Instead, problem instance b) gives rise to a mathematical programming with 120 continuous variables, whose solution, by the same commercial software as above and on the same platform, requires less than one second.

\section{CONCLUSIONS AND FUTURE DEVELOPMENTS}

The paper presents a discrete event approach to the optimal scheduling of charging operations of electric vehicles. The effectiveness of the proposed approach has been tested by a real case study, considering a daily time horizon. Future developments will regard the definition of strategies that involve also the sequencing of services and not only their timing. Finally, the case of allocation of services to multiple charging stations, will be investigated.

\section{REFERENCES}

[1] N. Shaukat, B. Khan, S. Ali, C. Mehmood, J. Khan, U. Farid, M. Majid, S. Anwar, M. Jawad and Z. Ullah, "A survey on electric vehicle transportation within smart grid system", Renewable and Sustainable Energy Reviews, vol. 81, pp. 1329-1349, 2018.

[2] S. Xu, Z. Yan, D. Feng and X. Zhao, "Decentralized charging control strategy of the electric vehicle aggregator based on augmented Lagrangian method", International Journal of Electrical Power \& Energy Systems, vol. 104, pp. 673-679, 2018.

[3] F. Laureri, L. Puliga, M. Robba, F. Delfino and G. Odena Bulto, "An optimization model for the integration of electric vehicles and smart grids: Problem definition and experimental validation", 2016 IEEE International Smart Cities Conference (ISC2), pp. 1-6, 2016.

[4] Z. Liu, Q. Wu, S. Huang, L. Wang, M. Shahidehpour and Y. Xue, "Optimal Day-Ahead Charging Scheduling of Electric Vehicles Through an Aggregative Game Model", IEEE Transactions on Smart Grid, vol. 9, no. 5, pp. 5173-5184, 2018.

[5] C. Chen, L. Xiao, S. Duan and J. Chen, "Cooperative Optimization of Electric Vehicles in Microgrids Considering Across-Time-and-Space Energy Transmission", IEEE Transactions on Industrial Electronics, vol. 66, no. 2, pp. 1532-1542, 2018.

[6] H. Fan, C. Duan, C. Zhang, L. Jiang, C. Mao and D. Wang, "ADMMBased Multiperiod Optimal Power Flow Considering Plug-In Electric Vehicles Charging", IEEE Transactions on Power Systems, vol. 33, no. 4, pp. 3886-3897, 2018.

[7] S. Bagher Sadati, J. Moshtagh, M. Shafie-khah, A. Rastgou and J. Catalão, "Operational scheduling of a smart distribution system considering electric vehicles parking lot: A bi-level approach", International Journal of Electrical Power \& Energy Systems, vol. 105, pp. $159-178,2018$.

[8] F. Kong, Q. Xiang, L. Kong and X. Liu, "On-Line Event-Driven Scheduling for Electric Vehicle Charging via Park-and-Charge", 2016 IEEE Real-Time Systems Symposium (RTSS), pp. 69-78, 2016.

[9] A. Di Giorgio, F. Liberati and S. Canale, "Electric vehicles charging control in a smart grid: A model predictive control approach", Control Engineering Practice, vol. 22, pp. 147-162, 2014.

[10] P. Palensky, E. Widl, M. Stifter, A. Elsheikh, "Modeling intelligent energy systems: Co-simulation platform for validating flexibledemand EV charging management », IEEE Transactions on Smart Grid, v 4, n 4, p 1939-1947, 2013.

[11] G. Ferro, F. Laureri, R. Minciardi and M. Robba, "An optimization model for electrical vehicles scheduling in a smart grid", Sustainable Energy, Grids and Networks, vol. 14, pp. 62-70, 2018.

[12] S. French, Sequencing and scheduling. Chichester: Wiley, 1987.

[13] F. Delfino, G. Ferro, M. Robba and M. Rossi, "An architecture for the optimal control of tertiary and secondary levels in small-size islanded microgrids", International Journal of Electrical Power \& Energy Systems, vol. 103, pp. 75-88, 2018. 